\title{
THE OCULAR COMPLICATIONS OF DIABETES
}

\author{
By P. D. Trevor-Roper, M.A., M.B., B.Chir., F.R.C.S. \\ Assistant Ophthalmic Surgeon, Westminster Hospital; Curator, Department of Pathology, Institute of Ophthalmology
}

Diabetes is a frequent source of blindness, accounting for 6 per cent. of the total who are registered as blind in England, and for 13 per cent. among the over-fifties, largely as a consequence of retinopathy and its culminating vitreous haemorrhage (Sorsby, 1956). And it is to the ophthalmologist that the diabetic so often presents with his first symptom of a little blurring of vision, or on routine ophthalmoscopy of the symptom-free.

The ocular manifestations of diabetes are strangely profuse, and their exact pathogenesis is little understood. But in the main they derive from primary changes in the blood-vessels (retinopathy, rubeosis, haemorrhage), nutritional upset (cataract), and the rather loosely conceived 'lowering of resistance' by which the various neurites and uveites are abetted. Of these the specifically diabetic lesions-the retinopathy and juvenile cataract demand our prime consideration.

\section{Retinopathy}

Diabetic retinopathy affects females more often than males, and its highest incidence is over the age of 50 - simply because of its long latent-period, and because diabetes is less common in the younger age-groups. Retinopathy generally follows Io to 20 years after the onset of the diabetes, and after 25 years the percentage of diabetics showing retinal changes is at least 75 per cent.; so with the increasing life-span of the population, and with the survival of the young diabetics (who in pre-insulin days would have died long before their retinopathy had time to emerge), there has been a steadily increasing incidence in retinopathy over the past decades. The incidence of the retinopathy is probably enhanced by the severity of the diabetes (although many dispute this), and by any coincident vascular disease; but it has no direct association with the severity of the symptoms or with the need for, the use of, or the abuse of insulin. So that the retinopathy (like diabetic cataract) tends to arise in those cases which have been undiagnosed for a long time, because the symptoms were slight, or because a high renal threshold had thwarted the tell-tale glycosuria. It seems to be a cardinal feature of the insulin-

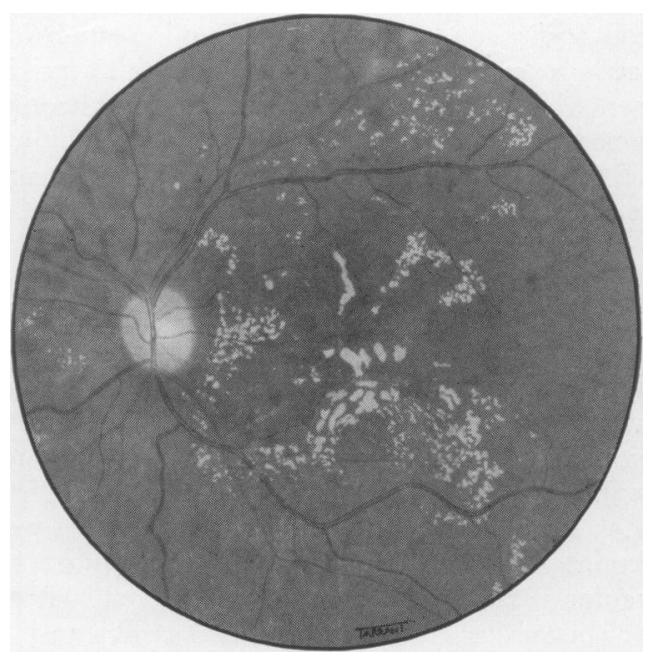

FIG. I.-Diabetic retinopathy (from Whittington, Proc. Roy. Soc. Med., 44, 743).

resistant type of diabetes which is probably derived from a pituitary-adrenal overaction rather than a pancreatic insufficiency, but it has recently been reported in cases of chronic pancreatitis and haemochromatosis (Ashton, 1958). Retinal arteriosclerosis and renal damage may well co-exist in these elderly diabetics, and their corresponding retinal changes may then augment those due to the diabetes, but this is only a chance overlap of two quite separate disease-processes within the same retina.

Although essentially bilateral, one eye is often weeks or months in advance of its fellow, and throughout its subsequent course the damage may differ widely on the two sides.

The ophthalmoscopic appearances of diabetic retinopathy are generally so distinctive that there is little cause for confusion with the retinopathies of arteriosclerosis and renal disease, although all three present with an assortment of haemorrhages, ' exudates,' and to a lesser extent with alterations in the retinal vessels.

The earliest imprints are minute localized varicosities on the retinal capillaries, which appear dispersed over the posterior pole of the eye; they 
are known as micro-aneurysms, and of a size just within the limits of ophthalmoscopic visibility. These are soon succeeded by small dark haemorrhages affecting the same region, and later they are joined by an occasional flame-shaped haemorrhage, resulting in the characteristic ' dot and blot' appearance.

These larger haemorrhages still tend to encircle the macula, and rarely lie in the immediate vicinity of the disc; occasionally they agglomerate nearer to the retinal periphery, when a small venous thrombosis can usually be found responsible, elsewhere the retina appears normal with a striking absence of oedema. The larger, irregular haemorhages tend to come and go, after the fashion of such superficial 'flame-shaped' haemorrhages, but the small 'blots' and their antecedent microaneurysms naturally remain fairly constant.

Changes in the retinal arterioles may be marked in the later stages, when arteriolar hyalinization and obliteration lead to total atrophy of the neighbouring capillary bed, and at the same time new vessels are often formed, sprouting from the venous side of the capillary network (Ashton, I953).

As time goes on, the characteristic waxy ' exudates' begin to appear in the same perimacular region, first as clusters of small specks, then becoming yellower as they grow, and revealing their classical hard and irregular outlines, but never attaining the size of the snow-bank exudates of renal retinopathy, although they may coalesce to form an archipelago skirting the macula like a circinate retinopathy. Pigmentary changes are always slight or absent.

Although these lesions always progress, many years may pass before the macula is involved, and only then may the patient become aware of any visual loss. However, at any time this tranquil course can be interrupted by a sudden severe haemorrhage, and if this irrupts into the vitreous, a dramatic blindness follows. These subhyaloid or vitreous haemorrhages will generally clear away in a few weeks, but often incompletely, especially where the underlying retinopathy is far advanced. Where new-vessel tufts have already formed into the vitreous the outlook is grave, for further haemorrhages are increasingly liable to follow. Then, as the blood-clot becomes organized, the tough fibrous stands of a 'retinitis proliferans' will be left straddling the retinal surface, and finally pull away the disorganised retina as a frank detachment; and the vision is thereafter remorselessly destroyed. Sometimes the sprouting of new vessels, may produce a vascular network that wafts about in the vitreous like fronds of seaweed, and well merits the name of ' rete mirabile,' while the veil-like sheet connecting these vessels

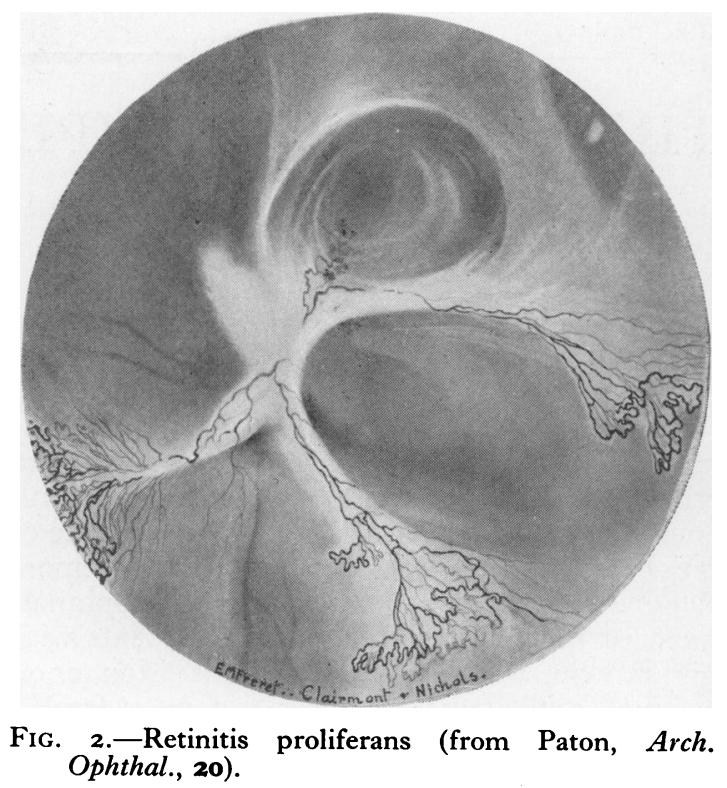

often simulates a detached retina; but subsequent bleeding from the surface of the veil produces a coating of fibrous tissue, so that the more typical picture of retinitis proliferans is the ultimate sequel.

Another, less familiar, stigma of diabetic $\frac{0}{0}$ retinopathy is seen in the retinal veins, which tend to form localized sausage-shaped distensions, dark in colour and about $\frac{1}{2}$ to I disc-diameters in length, over any part of the retina except the periphery and the immediate surround of the optic disc. These swollen veins generally herald a progesssive lesion, and they may be the first fundus change when the retinopathy starts in adolescents or young adults. From them new venules may arise and a vitreous haemorrhage may develop even before the other characteristic features of the retinopathy are manifest. Later these fusiform enlargements may become distorted into loops, and varicosities, which are further complicated by anastomoses with neighbouring venous loops or their branches.

The pathogenesis of these microaneurysms, which form the cardinal lesion of the diabetic retina, is yet imperfectly understood. The only established site outside the retina in which similar capillary dilatations are found is the renal glomerulus in cases of intercapillary glomerulosclerosis (Kimmelsteil-Wilson disease) (Ashton, 1949) in which indeed there is always, if only microscopically, an associated retinopathy and there may be the same underlying cause.

The microaneurysms (and the small, blotshaped haemorrhages that succeed them) form within the deeper layers of capillaries (within the 
outer molecular retinal layer), where these capillaries are most abundant - at the posterior pole of the eye; and this capillary network is itself most dense around the venae efferentes. The localizing factor in the production of these microaneurysms appears to be a partial venous stasis and a fatty infiltration of the endothelium of the capillaries and venules, while a fatty deposit encircling their walls prompts the actual aneurysmformation.

As the disease advances, the arterial side of the circulation also becomes involved; hyalinization of the terminal arterioles and pre-capillary vessels of the retina leads to narrowing and gradual occlusion of their lumina; and then new dilated channels form within the capillary bed, which eventually atrophies completely when the arterioles become totally occluded. From the neighbourhood of these atrophic areas new vessels may bud out into the vitreous in an abortive attempt to re-establish the circulation (hence the ' rete mirabile'). The majority of the aneurysms arise through a degeneration within a capillary loop, but the basic cause remains unknown, although it has been suggested that it is due to involvement of the muco-polysaccharide protein complexes of the vessel wall. Small isolated micro-aneurysms may be found in the non-diabetic subject (in some apparently healthy eyes, thrombotic glaucoma, choroido-retinitis, etc.); nevertheless the lesions are highly characteristic of diabetes and their presence should always suggest the diagnosis.

The 'exudates' also develop within this same deep capillary plexus, and are either fatty or hyaline in composition, and are presumably also the legacy of these combined factors of venous stasis and fatty degeneration of the capillary wall.

The treatment of diabetic retinopathy is largely restricted to providing moral support as the vision is gradually eclipsed. The diabetes must, of course be carefully controlled, and among the very large number of investigators, the majority believe that this will significantly delay the onset and the progress of the retinopathy (Ashton, r958). A regulated life, designed to avoid a sudden venous congestion or rise of arterial pressure will, at any rate theoretically, help to avoid unnecessary rupture of the thin-walled microaneurysms, and repeated small doses of $\mathrm{X}$-rays have also been recommended to inhibit the mitosis of the capillary endothelial cells, from which the new capillaries sprout (Bignell, I957; Kurrle, I957). Various systemic treatments have evoked transient enthusiasm, such as Rutin, but are now largely discredited. An association of retinopathy with adrenal overactivity was noted by Becker (I952) and later with deficiency of cyanocobalamin or 'vitamin B.12' (Becker, Lang and Chow, 1953), which may itself be due to an increased B.I2 excretion provoked by adrenal overactivity, so treatment with intramuscular doses of B.12 have recently been tried with questionable success (Sancetta et al., I95I; Cameron and Ahern, 1958). As a drastic intervention hypophysectomy has also its advocates in carefully selected cases (Luft, I955).

Lipaemia retinalis presents a singular fundus picture without any effect on the patient's vision. It may be seen when the lipoid content of the blood is over 5 per cent., and it chiefly arises in young patients with a marked acidosis (an increasing rarity in these post-Insulin days). The bloodcontent of both arteries and veins varies from a creamy colour at the periphery to a salmon-pink at the disc, the whole of the retina appearing rather matt and ill-defined. The fundus picture becomes completely normal within a few days of insulin and carbohydrate treatment.

\section{The Lens}

A sudden alteration in the volume of the lens is a frequent finding among diabetics, and this necessarily causes a change in refraction, which may be very transient, but is sometimes very dramatic. The lens capsule is semipermeable, so in the acute stage of diabetes the fluid content of the lens promptly increases, and myopia ensues; but as soon as diet or insulin have controlled the hyperglycaemia, the process is reversed, the lens shrinks, the myopia is shed, and several degrees of hypermetropic may even result.* The unstable diabetic will thus have an equally unstable focal range, and such patients must be sight-tested only at periods of a balanced blood-sugar, or the spectacles will be far from correct. In extreme cases of lens shrinkage, its capsule may be thrown into folds; and these can sometimes be moulded by pressure on the eyeball itself if the whole eye is very soft (as in diabetic coma).

The true Diabstic Cataract occurs only in youth, generally between the age of $\mathrm{r}_{5}$ and 25 , but exceptionally in infancy; and it is remarkable for its rapidity, the whole lens becoming opaque within a few weeks in adolescence, or within a few days in children. Its development is roughly proportional to the severity of the diabetes,

\footnotetext{
* This apparent paradox of the lens swelling in hyperglycaemia and vice-versa is frequently misunderstood, since the raised sugar content in the aqueous might be expected to raise rather than lower its osmotic pressure. In fact, the hyperglycaemic blood is promptly diluted by the tissue fluids, and an increased excretion follows of a urine with high salt content and osmotic pressure, which is replaced volumetrically by drinking water. Hence the blood volume increases and its salt content decreases, both of which promote a lowered aqueous osmotic pressure (Duke Elder, 1925).
} 


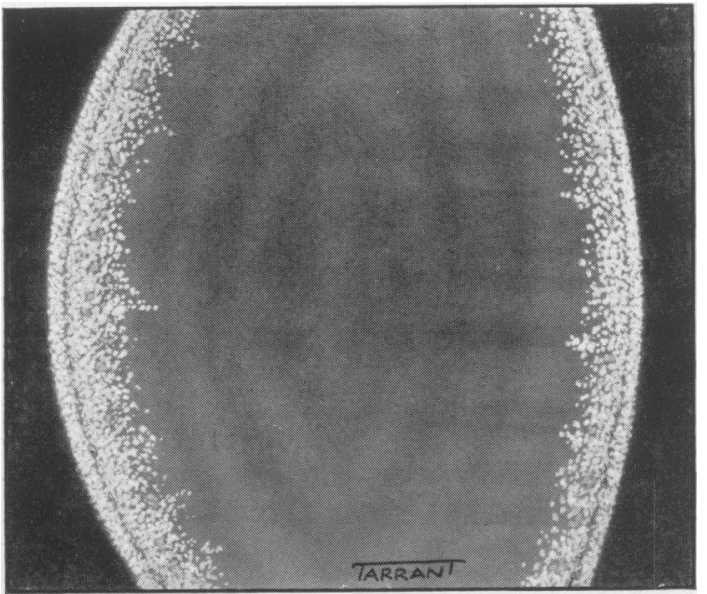

FIG. 3.-True diabetic cataract. Optical section showing characteristic subcapsular flakes.

although the exact chemical factors are not clearly known, and it is a less frequent finding with the effective modern therapy. The early stages are morphologically quite characteristic, for a blanket of milk-white 'snow-flakes' collect just beneath the anterior and posterior lens capsules, interspersed with iridescent vacuoles and clefts. Gradually these flocculent opacities coalesce, and the whole lens becomes smoky in appearance, and ultimately homogeneous and creamy when the cataract is complete. Only the earliest clouding of the lens, which is mainly due to hydration, is reversible; for once the lens protein has coagulated and agglutinated, it can never clarify again; and, although treatment may brake its progress, a a complete cataract is always the final outcome.

Such diabetic cataracts can be removed surgically without any special difficulties. Since both eyes are normally affected at the same time, there is an obvious need to restore useful sight without delay, so that a 'linear extraction' is preferred to dilatory ' needlings'; the second cataract should be removed at the earliest convenience after fitting the first eye with its aphakic spectacles.

A simple senile cataract arises in the elderly diabetic with slightly greater frequency than in the population at large. Such lens opacities often start beneath the posterior capsule, with a brassy iridescence reminiscent of the more brilliant ' polychromatic lustre' in ' complicated' cataracts (i.e. secondary to a uveitis) which similarly start beneath the posterior capsule. This early appearance is often held to justify labelling such senile cataracts also as being specifically diabeticmuch less singular than the 'true diabetic cataract' of youth, but at any rate vastly more common; but since they behave in every other respect like the familiar senile cataract, and present no special idiosyncrasies when they come to be extracted, their diabetic label has no practical relevance; (at their extraction, a few special considerations do weigh in regard to the diabetic eye rather than to the lens itself, for such eyes are more liable to a complicating haemorrhage, while the prognosis must be more guarded, si nce a diabetic retinopathy may well be concealed behind the opaque lens, and the ultimate vision proportionately poor). Very rarely the adult diabetic lens may develop a mild 'opacity' due simply to hydration, which is to that extent reversible, as with the similar hydration-opacities of the true diabetic cataract in its earliest stages.

\section{The Iris}

Rubeosis Iridis is the most important diabetic iridopathy; it is infrequently seen, since it generally betokens a severe diabetes with secondary cardio-renal damage, and young patients are thus principally affected. A meshwork of new blood-vessels appears over the pupillary zone and over the root of the iris, with several connecting vascular trunks spanning the relatively avascular middle-zone: this wreath of intertwining vessels encircles the pupil (but not transgressing itg of margin), and is readily seen with the naked eye while those vessels encircling the iris-root dise appear from sight as they sweep across the angle of the anterior chamber, forming peripheral anterior synechiae. Sooner or later glaucoma supervenes, either as a direct consequence of the impediment to aqueous drainage caused by the $\overline{\bar{O}}$ peripheral anterior synechiae, or more usually from the haemorrhages that follow rupture of these new thin-walled vessels. This glaucoma admits little effective treatment, since any incision into the eye will almost certainly induce further hyphaemas or vitreous haemorrhages, and indeed a retro-bulbar injection of alcohol to relieve the pain is generally all that can be usefully done as the sight is destroyed.

A scattering of iris pigment is the other familiar 윽 clinical feature of the diabetic iris, but of little $\frac{D}{O}$ pathological significance. It is due to an oedema of the pigmented posterior layer of the iris, N abetted by a glycogenous infiltration (which indeed may also involve the mesodermal layers); these swollen cells vacuolate and break, so that the liberated pigment granules may be found adhering to the anterior lens capsule, the posterior corneal surface, and even colouring the aqueous.

\section{Ocular Inflammations}

In the presence of an established diabetes there is an added liability to inflammation in all of the $\stackrel{?}{\mathbb{D}}$ various ocular tissues. Thus there is said to be 
an increased incidence of anterior uveitis, keratitis and conjunctivitis; the former (which often takes the form of an 'acute iritis') is said to be characterized by an unusual degree of oedema and injection both circumcorneally and of the iris itself; and by its tendency to produce a gelatinous exudate in the aqueous.

An optic neuritis, generally in the form of a retro-bulbar neuritis rather than a papillitis, is again relatively common in diabetics; probably because the metabolic upset simply renders such patients susceptible to a neuritis from coincident toxins (such as tobacco and alcohol), which they would normally be amply able to withstand. Such a neuritis is transient and bilateral, and presents with a dense central or paracentral scotoma.

In the same category fall the various peripheral neurites to which diabetics are notoriously subject, and which may well involve any of the nerves to the extra-ocular muscles-particularly the abducent nerve. Such palsies, with their consequent diplopia, generally recover within two or three months. They are usually monocular, and it must be remembered that in the ageing diabetic, an abducent palsy is a relatively common sequel simply to their coincident cardiovascular disease. Similarly the ciliary muscle may be affected, with a transient weakness of accommodation as its legacy.

\section{BIBLIOGRAPHY}

ASHTON, NORMAN (1949), Brit. F. Ophthal., 33, 407.

ASHTON, NORMAN (1953), Ibid., 37, 282.

ASHTON, NORMAN (1958), Advanc. Ophthal., 8, r.

BECKER, B. (1952), Amer. Intern. Med., 37, 273.

BECKER, B., LANG, C. A., and CHOW, B. F. (1953), f. clin. Nutrit., $\mathrm{I}, 4 \mathrm{I} 7$.

BIGNELL, J. L. (1957), Trans. ophthal. Soc. Aust., 17, 58.

CAMERON, A. J., and AHERN, G. J. (1958), Brit. F. Ophthal., 42,686 .

DUKE ELDER, S. (1925), Ibid., 9, 167.

KURRLE, G. (1957), Trans. ophthal. Soc. Aust., 17, 6r.

LUFT, R., OLIVECRONA, H., IKKOS, D., KORNERUP T., and LJUNGGREN H. (1955), Brit. med. $\mathscr{F}$., ii, 752 .

SANCETTA, S. M., AYRES, P. R., and SCOTT, R. W. (195I), Ann. Intern. Med., 35, 1028.

SORSBY, A. (1956), 'Blindness in England,' London. H.M Stationery Office.

\section{HEPATIC DISEASE}

(Postgraduate Medical Journal)

Price: 4s. Od. post free

\section{JAUNDICE}

Sheila Sherlock, M.D., M.P.C.P.

\section{HEPATIC COMA}

J. M. Walshe, M.A., M.R.C.P.

SURGICAL TREATMENT OF PORTAL HYPERTENSION

A. I. S. Macpherson, $\mathrm{Ch}$ M., F.R.C.S.E.

WILSON'S DISEASE

A. G. Bearn, M.D.
ASCITES IN LIVER DISEASE

Michael Atkinson, M.D. (Lond.)

M.R.C.P.

PSYCHIATRIC ASPECTS OF LIVER

DISEASE

Esther A. Davidson, M.R.C.P.Ed., and

W. H. J. Summerskill, M.A., M.R.C.P.

PERCUTANEOUS PORTAL

VENOGRAPHY

David Sutton, M.D., M.R.C.P., F.F.R.

Published by

THE FELLOWSHIP OF POSTGRADUATE MEDICINE

60, Portland Place, London, W.1 